\title{
Editorial
}

\section{Suicide risk assessment: time to think again?}

\author{
J. V. Lucey ${ }^{1}$ and B. Matti ${ }^{2}$ \\ ${ }^{1}$ Psychiatry, TCD, Dublin, Ireland and ${ }^{2}$ Psychiatry, SPMHS, Dublin, Ireland
}

\begin{abstract}
This paper considers recent research on suicide risk assessment to support calls for a 'rethink' of our assessment of the patients in our care, along with the adoption of a more collaborative approach to care planning with service users who remain at risk of self-harm and in need of a plan for their safety.
\end{abstract}

Keywords: Safety; suicide risk assessment; risk factors

(Received 12 August 2021; revised 20 October 2021; accepted 4 November 2021)

\section{Introduction}

Globally, an estimated 703000 people die by suicide every year. There are more deaths annually worldwide due to suicide than those due to Malaria, HIV/AIDS, breast cancer, or war and homicide. More than one in every hundred deaths across the world results from suicide (WHO, 2021).

Comparison of the Irish (ROI) suicide rate with the rest of Europe is based on figures produced by Eurostat. The latest of these comparisons relates to 2017. In that year, the ROI suicide rate of 8.4 per 100000 population was ninth lowest of 33 European countries (National Office for Suicide Prevention, NOSP, 2020). In 2020 in Ireland (ROI), 340 lives were lost to suicide, (81 were females and 259 were males). Suicide in ROI in 2020 occurred at a rate of 6.8 per 100000 per annum.

Rates of suicide at a population level have fallen in Ireland (ROI). Although NOSP advises against drawing conclusions based on year-on-year comparisons, the rate of suicide in males fell throughout the past decade from 22.4 (per 100 000) in 2001 to 10.5 (per 100000 ) in 2020. There were 541 'registered' suicides in 2012, but by 2020 the total number of suicides (males and females) was down to 340 . The number of suicides in Ireland (ROI) continued to fall from 2015 to 2020 (NOSP, 2020), the years covered by the government of Ireland national strategy for prevention of suicide entitled 'Connecting For Life' (www.gov.ie) (Government of Ireland Publications, 2015).

Measures taken at a population and sub-population level (such as those delineated in Connecting for Life) can reduce rates of suicide in the community. The WHO notes that such national programmes are most likely to be effective when they include four types of action, limiting access to lethal means of suicide (e.g. pesticides, firearms, and certain medications), improving interaction with the media to promote responsible reporting of suicide, fostering socio-emotional skills in young people and adolescents, and

Address for correspondence: J. V. Lucey, Psychiatry, TCD, Dublin, Ireland. (Email: jlucey@stpatsmail.com)

Cite this article: Lucey JV and Matti B. Suicide risk assessment: time to think again? Irish Journal of Psychological Medicine https://doi.org/10.1017/ipm.2021.76 earlier identification, assessment, and management of those who exhibit suicidal behaviours (WHO, 2021). Clearly, the work of all those involved in suicide reduction in our community, including the NOSP, must continue if the rate of suicide in Ireland is to decline further (WHO, 2012).

The best clinical practice aims not only to reduce suicide in the community but also to prevent it among patients in clinical care (NICE, 2018). It is not clear how well current risk management contributes to this latter goal. Collecting data on this point is not easy. Rates of suicide in clinical settings are lower than those rates in the community and only a minority of patient suicides involve in-patients. According to the UK National Confidential Inquiry into Suicide and Safety in Mental Health (Appleby et al. 2019), suicides by in-patients account for less than $6 \%$ of all patient deaths. Recent data revealed most patients who died by suicide in the UK had been assessed as low risk in their last contact with mental health services (Graney et al. 2020). It seems likely that the same is true in Ireland.

Risk assessment is a standard part of clinical practice, but service users have expressed frustration with clinical contacts that are 'too focused on assessing risk and not enough on assessing needs' (Anonymous, 2020). Dissatisfaction has also been articulated by clinicians concerned about the burden of psychiatry and its effect on clinicians' mental health (Mac Suibhne et al. 2017). There have been calls for a complete 'rethink' of psychiatry's attitude and response to patient suicide (Lancet Psychiatry, 2020).

The first step towards a 'rethink' is an empirical one (Runeson et al. 2017). Such an examination reveals that traditional risk assessment has not been as effective at suicide prediction as previously believed. The tools used in this risk assessment are unsatisfactory. In any case, there is more to the management of suicide than the assessment of risk. Revised forms of clinical practice are needed (NICE, 2011). The hope is for a more integrated, collaborative, and comprehensive assessment of at-risk patients. This will require individualised care planning around two inter-related areas, patient 'risk' and patient 'safety'.

A 'rethink' includes an acknowledgement of the limitations of traditional risk assessment and a consideration of a more sustainable approach to the assessment of safety. These two things 
(assessment of risk and assessment of safety) need to be examined together and in more detail.

\section{Assessment of risk}

Traditional suicide risk assessment is focused on the identification of clinical factors associating suicidal ideation with suicide (Arensman et al. 2019). These factors include male gender, a previous history of suicidal thoughts and behaviours, trauma, significant physical illness, alcohol or substance misuse - intoxication or withdrawal, mental illness, a family history of suicide, loneliness, and access to lethal means of self-harm (Beattie \& Devitt, 2015). It is now clear that mathematical summation of these correlates cannot generate a useful predictive tool for suicide.

The UK National Institute for Health and Care Excellence (NICE) advises that tools used for risk assessment should not be used as predictive measures and should not be regarded as such (NICE, 2011). The low frequency of patient suicide places 'an absolute ceiling' on the development of data with sufficient predictive ability. Any numbers that emerge are without sufficient sensitivity and specificity, and without positive or negative predictive value. Consequently attempts to design instruments for the prediction of suicide have been described as 'misleading and potentially harmful' (Carter \& Spittal, 2018).

Methods used to stratify suicide risk into broad clinical categories have also been unsuccessful. Traditionally risk factors belong to three inter-related categories. The first is a group of correlates known as (a) 'static' factors. These are associated with suicidal thoughts and behaviours. Examples of 'static' factors include age or male gender. Unfortunately, these 'static' factors make poor predictors of suicide and ineffective targets for risk reduction.

In the second category are correlates referred to as (b) 'dynamic' risk factors. These have been used in attempts to stratify the population into 'high' and 'low' risk groups for suicide. An example of a 'dynamic' risk factor is 'loneliness'. Unfortunately, these 'dynamic' correlates are also poor predictors of suicide and even less effective targets for treatment (NICE, 2018). Stratification of patients with suicide risk on the basis of 'dynamic' factors has not proved useful. Meta-analysis of 50 years of research in the US found no broad category or sub-category of suicidal thoughts or behaviours accurately predicted suicide above chance (Franklin et al. 2017).

A third group of correlates may be more useful in the management of suicide risk. These correlates are known as (c) 'causal' risk factors, and they are associated with interventions likely to reduce the probability of a suicide. 'Causal' risk factors are potentially modifiable treatment targets, and so they are more likely to be protective against suicide. Examples of 'modifiable' or 'causal' factors in the community include physical illness, intoxication/withdrawal, mental illness, and access to lethal means (Franklin et al. 2017). Examples of 'causal' factors in the in-patient setting include levels of supervision offered to patients on admission to hospital, removal of ligature points from in-patient wards, and prevention of absconding from those wards.

\section{Assessment of safety}

Suicides are preventable (Parekh \& Phillips, 2014). Effective initiatives need to be 'comprehensive, integrated, and synergistic' since 'no single approach can impact alone on an issue as complex as suicide'. Effective multisectoral prevention measures include restrictions on access to the most common means, earlier and more effective management of mental disorders and substance misuse disorders, and better support for vulnerable individuals, including better follow-up care and more effective campaigns to fight stigma and provide support for those bereaved by suicide (WHO, 2012).

The burden of responsibility for safety carried by those caring for people at risk of suicide is a heavy one. It is time to acknowledge 'the reality of suicide risk assessment' (Lancet Psychiatry, 2020) mindful of the rights of service users and the expectations placed upon clinical staff and their health care organisations. It is time to develop a more collaborative clinical model based around safety and not only risk.

Listwise risk assessments have not been helpful for patients or for clinicians. Over time the service user comes to understand that he/she cannot access 'therapy' unless deemed 'low risk' and so inevitably the patient becomes less open about their true feelings (Anonymous, 2020). After a patient's suicide, the emotional burden experienced by staff in mental health services amplifies the personal, professional, and legal stresses already born by them. Few if any of these pressures are mitigated by traditional 'risk' assessment. A 'blame culture' adds further trauma to the substantial load already carried by psychiatric staff (Mac Suibhne et al. 2017).

Key opinion formers have tried to counter the risk/blame culture with arguments in favour of a more collaborative approach to risk assessment (Szmukler et al. 2019) but the most effective response will come through changes in clinical practice. Re-defining the idea of 'safety' rather than 'risk' is a work in progress but practice guidelines are emerging. The UK NICE has published one such guidance document entitled 'Self-harm in over 8s: long term management' (NICE, 2016). This detailed document runs to more than 30 pages and recommends an integrated comprehensive psychosocial assessment of risks and needs for suicidal and at-risk persons. It encourages staff to understand and to engage with people who are at risk of self-harm in order to initiate and maintain a more therapeutic relationship and so to work towards a meaningful 'safety plan'.

A reduction of 'causal' risk factors within services would go some way towards improving safety and reducing suicide risk. Leadership bodies have re-stated the evidence based view that psychiatrists should not use risk assessment tools or scales to predict future suicide or repetition of self-harm, but the reality is that these risk assessment tools are still in widespread use (Anonymous, 2020). It is becoming clear that the way forward for assessment goes beyond the issue of improving prediction and towards progress with 'safety planning'. Therapeutic collaboration will include the development of 'self-management safety strategies' more helpful to those at suicide risk.

A cognitive focus on safety rather than risk led to the development of these safety planning-type interventions. Intended for those at imminent risk they enable the construction of an agreed set of coping strategies and supports within the individual care plan (Stanley \& Brown, 2012). Recent meta-analyses of these safety planning interventions suggest that they can reduce the risk of suicidal behaviour by $43 \%$ (where the number needed to treat is 16 ; Nuij et al. 2021).

Interventions such as these are especially effective when are supported by mental health services genuinely addressing the needs of at-risk individuals. These services provide increased access to measures likely to enhance treatment of associated mental health problems and difficulties. The concept of suicide risk assessment is moving on, becoming less of a guessing game and more of a collaborative therapeutic intervention focused on safety first. A shift in this direction enables an alliance that service users are calling for and that service providers can sustain. 


\section{Conclusion}

The aim of health services is to reduce suicide at a population level and to eliminate it at a clinical level (WHO, 2012). Clinicians through their training possess the ability to collaborate with their patients. Promoting this therapeutic alliance is the best way forward for suicide risk management in the clinical context. Practice guidelines around suicide risk management and safety planning could be developed and evaluated in Ireland. It is likely that such clinical guidance on suicide risk management would include at least three recommendations, (1) an end to the reliance on risk prediction, (2) the elimination of 'causal' risks for suicide in clinical settings, and (3) the promotion of better patient safety using individualised safety planning-type interventions.

Changes in clinical practice such as these will take time (Herzlinger, 2006) since clinical advances often lag behind the evidence base (Kristensen et al. 2016), but changes in teaching and training at undergraduate and postgraduate levels will help to progress this re-orientation of our clinical approach by moving our suicide risk management further towards safety planning.

It is time to 'rethink' our practice of suicide risk assessment. A new safety approach will be more compassionate to our patients, more helpful to our staff, and more sustainable for the future of our services.

\section{Conflicts of interest. None.}

Ethical standards. The authors assert that all procedures contributing to this work comply with the ethical standards of the relevant national and institutional committee on human experimentation with the Helsinki Declaration of 1975, as revised in 2008 .

Financial support. This research received no specific grant from any funding agency, commercial or not-for-profit sectors.

\section{References}

Anonymous (2020). The reality of risk assessment. Lancet Psychiatry 7, 1003-1004.

Appleby L, Kapur N, Shaw J, Hunt IM, Ibrahim S, Turnbull P, Bojanić L, Rodway C, Tham S-G, Richards N, Burns J (2019). The National Confidential Inquiry into Suicide and Safety in Mental Health. Annual Report: England, Northern Ireland, Scotland and Wales. University of Manchester.

Arensman E, Larkin C, McCarthy J, Leitao S, Corcoran P, Williamson E, McAuliffe C, Perry IJ, Griffin E, Cassidy EM, Bradley C, Kapur N, Kinahan J, Cleary A, Foster T, Gallagher J, Malone K, Ramos Costa AP, Greiner BA (2019). Psychosocial, psychiatric and work-related risk factors associated with suicide in Ireland: optimised methodological approach of a case-control psychological autopsy study. BMC Psychiatry 19, 275.

Beattie D, Devitt P (2015). Suicide: A Modern Obsessions. Liberties Press Publisher: Dublin, pp. 2-30.
Carter G, Spittal MJ (2018). Suicide risk assessment: risk stratification is not accurate enough to be clinically useful and alternative approaches are needed. Crisis 39, 229-234.

Franklin JC, Ribeiro JD, Fox KR, Bentley KH, Kleiman EM, Huang X, Musacchio KM, Jaroszewski AC, Chang BP, Nock MK (2017). Prediction of suicide using factors: a meta-analysis of 50 years of research. Psychological Bulletin 143, 187-232.

Government of Ireland Publications (2015). Connecting for Life: Ireland's National Strategy to Reduce Suicide 2015 - 2020. Department of Health. Health Services Executive (HSE) (2015) Connecting For life. Department of Health (DoH). http://hdl.handle.net/10147/572879

Graney J, Hunt IM, Quinlivan L, Rodway C, Turnbull P, Gianatsi M, Appleby L, Kapur N (2020). Suicide risk assessment in UK mental health services: a national mixed-methods study. Lancet Psychiatry 7, 1046-1053.

Herzlinger RE (2006). Why innovation in health care is so hard. Harvard Business Review 84, 58-66, 156.

Kristensen N, Nymann C, Konradsen H (2016). Implementing research results in clinical practice - the experiences of healthcare professionals. BMC Health Services Research. 16, 48. doi: 10.1186/s12913-016-1292-y.

Lancet Psychiatry (2020). Suicide and the psychiatrist. Lancet Psychiatry 7, 1001.

Mac Suibhne S, Chorcorain AN, Lucey JV (2017). Acknowledging mental illness in psychiatrists. International Journal of Social Psychiatry 63, 561-562.

NICE (2011). Guidance: Self-Harm - Longer Term Management - 'Do Not Use Risk Assessment Tools and Scales to Predict Future Suicide or Repetition of Self-Harm (G133)' National Institute for Health and Clincal Excellence UK Publisher, 22, 1.3.11.

NICE (2016). Self-harm in over 8s: short-term management and prevention of recurrence- NICE guideline CG16 and Self-harm in over 8s: long term management (2011) NICE guideline CG133 [Internet]. London: National Institute for Health and Care Excellence (UK). Available from: https:// www.ncbi.nlm.nih.gov/books/NBK552073/

NICE (2018). Preventing Suicide in Community and Custodial Settings. NICE UK Publisher, National Institute for Health and Clinical Excellence (NICE Guidance) (NG 105).

NOSP (2020). Annual Report - Suicide in Ireland. National Office for Suicde Prevention NOSP Ireland Publisher, pp. 68-73.

Nuij C, Van Ballegooijen W, De Beurs D, Juniar D, Erlangsen A, Portzky G, O'Connor RC, Smit JH, Kerkhof A, Riper H (2021). Safety planning-type interventions for suicide prevention: meta-analysis. British Journal of Psychiatry 219, 419-426.

Parekh A, Phillips M (2014). Preventing Suicide: A Global Imperative. WHO: Geneva.

Runeson B, Odeberg J, Pettersson A, Edbom T, Adamsson IJ, Waern M (2017). Instruments for the assessment of suicide risk: a systematic review evaluating the certainty of the evidence. PloS One 12, 12-17.

Stanley, B., \& Brown, G. (2012). Safety Planning Intervention: A brief intervention to mitigate suicide risk. Cognitive and Behavioral Practice, 19(2), 256-264.

Szmukler G, Richardson G, Owen G (2019). 'Rabone' and four unresolved problems in mental health law. The Psychiatrist 37, 297-301.

WHO (2012). Public Health Action for the Prevention of Suicide: A Framework. WHO: Geneva.

WHO (2021). Suicide Worldwide in 2019: Global Health Estimates. WHO: Geneva. 